\title{
Scaling Behavior of Multiplicity Distribution under First-Order Quark-Hadron Phase Transition
}

\author{
C.B. Yang ${ }^{1,2}$ and X. $\mathrm{Cai}^{1,3}$ \\ ${ }^{1}$ Institute of Particle Physics, Hua-Zhong Normal University, Wuhan 430079, the People's Republic of China \\ 2 Theory Division, RMKI, KFKI, Budapest 114., Pf. 49, H-1525 Hungary \\ Physics Department, Hubei University, Wuhan 430062, the People's Republic of China
}

(August 24, 2018)

\begin{abstract}
Multiplicity distribution in small bins is studied within the Ginzburg-Landau description for first-order quark-hadron phase transition. Direct comparison of the distribution with a Poisson one (with the same average) is made. Dynamical factor $d_{q}$ for the distribution and ratio $D_{q} \equiv d_{q} / d_{1}$ are studied, and novel scaling behaviors between $D_{q}$ are found which can be used to detect the formation of quark-gluon plasma.

PACS number(s): 05.70.Fh, 05.40.+j, 12.38.Mh

Key Words: Multiplicity distribution, phase transition, scaling behavior, Ginzburg-Landau description
\end{abstract}

It is well-known that high energy heavy-ion collision is the unique way to study the vacuum properties of quantum chromodynamics (QCD) in laboratory. In the collisions a hot new matter state, quark-gluon plasma (QGP), might be formed, and the system will cool with its subsequent expanding and will undergo a phase transition from deconfined QGP to confined hadrons. Since only the final particles in the collisions are observable in experiments, one may be asked to search for the signals about the phase transition from only those particles. Contrary to the final state leptons, which are produced mainly in the early stage of the new matter state and do not participate the strong interactions, hadrons are emitted out in the freeze-out process and may carry some relic information about the evolution of the system and the interactions. Up to now many possible signals about the phase transition, such as $J / \Psi$ suppression, strangeness enhancement, etc., have been proposed theoretically. But all of them can be reproduced from conventional physics. Thus, more further studies about the signal are needed.

In this Letter, we study the final state hadrons for the signal based on the Ginzburg-Landau description of the phase transition. Speaking generally, multiplicity distribution of final state hadrons is one of the most important and most easily accessible experimental quantities in high energy leptonic and hadronic collisions. The study of the global distribution, from the earlier well-known KNO scaling and its violation [1,2] to a recently novel scaling form [3], can give us a lot about the dynamical features of the multiparticle production processes. Local multiplicity distribution has also been studied for many years in terms of a variety of phase space variables [4], and substantial progress has been made recently in deriving analytical QCD predictions for those observables [5]. Based on assuming the validity of the local parton hadron duality hypothesis, these predictions in [5] for the parton level can be compared to experimental data. A global and local study of multiplicity fluctuations [6] shows, however, that those theoretical predictions have significant deviation from experimental data. The significant deviation of theoretical predictions from experimental data indicates that we know only a little about multiparticle production processes since the hadronization process in soft QCD is far from being understood, and phenomenological investigation may still be valuable.

It is well-known that an important feature associated with critical phenomena is large fluctuations. For multiparticle production processes the scaled factorial moments [7] is the most effective way to extract dynamical fluctuations. In fact, the moments are averages of some quantities over multiplicity distribution. Then one can see that the dynamical information for the processes can also studied in terms of the distribution. In particular, one may expect that whether the colliding system undergoes a quark-hadron phase transition will be reflected in the distribution. In this Letter we try to investigate multiplicity distribution in some small two-dimensional kinematic bin (which can be rapidity and transverse momentum or azimuthal angle, for example) in high energy heavy-ion collision processes. Because the final state hadrons may carry some relic information about their parent state, the investigation of the multiplicity distribution may be interesting and useful for probing the formation of QGP. As is shown in a previous work [8], the study can reveal more information than that of factorial moments can since the average over multiplicity in the calculation of the moments may conceal some useful information. In this Letter we are limited to discussing multiplicity distribution under the assumption of a first-order phase transition, within Ginzburg-Landau description for the phase transition. The multiplicity distribution for the case of second-order quark-hadron phase transition has been studied in [8], in which two new quantities are introduced to measure the influence on the distribution of dynamical fluctuations, and interesting scaling results are shown. It should be pointed out that the scaled factorial moments are studied within the same description for phase transitions by many authors for both second-order $[9,10]$ and first-order [11-14] phase transitions, and a universal scaling exponent $\nu \simeq 1.30$ is given in $[9,10,13,14]$. 
In Ginzburg-Landau theory, the multiplicity distribution turns out to be a Poisson distribution if the field is pure coherent. Conversely, the distribution turns into a negative binomial distribution if the field is completely chaotic. In reality, one can assume multiparticle production arising from a mixture of chaotic and coherent field, so the multiplicity distribution in real processes is not a Poisson one nor a binomial one, and the deviation of the distribution from a Poisson one is due to dynamical fluctuations. The real quantity concerned with the dynamical process is the relative deviation of $P_{q}$ (the probability with $q$ hadrons in a bin) from its Poisson counterpart $p_{q}$. Thus studying the relative deviation may reveal features of dynamical mechanisms involved. As introduced in [8], the relative deviation of $P_{q}$ from its Poisson counterpart $p_{q}$ can be measured by ratio $d_{q}=P_{q} / p_{q}$. The ratio $d_{q}$ can be called dynamical factor, since it is 1.0 unless there are dynamical fluctuations in the process. Dynamical fluctuations is shown to be exist if the ratio is far from 1.0, either much larger or much smaller than 1.0, for some $q$. For the definition of $d_{q}$ to make sense, it is necessary to let the mean multiplicity $\langle n\rangle$ for $P_{q}$ and $p_{q}$ be the same which will be given below. In Ginzburg-Landau description for first-order phase transition the distribution $P_{q}$ is given in [9]

$$
P_{q}(\delta)=Z^{-1} \int \mathcal{D} \phi p_{q}\left(\delta^{2}|\phi|^{2}\right) e^{-F[\phi]}
$$

where $Z=\int \mathcal{D} \phi e^{-F[\phi]}$ is the partition function, $p_{q}(\bar{s})$ is the Poisson distribution with average $\bar{s}, p_{q}(\bar{s})=\bar{s}^{q} / q ! \exp (-\bar{s})$, and $F[\phi]$ the generalized free energy functional for the first-order phase transition in a two-dimensional bin $\delta^{2}$

$$
F[\phi]=\delta^{2}\left[a|\phi|^{2}+b|\phi|^{4}+c|\phi|^{6}\right],
$$

with $b<0, c>0$, and $a \propto T-T_{C}$ representing the temperature. For more information about the discussion of the functional, see [8]. For different sets of parameters the system can be in the quark phase or hadron phase, see [11-14] for detail. In real experiments the temperature at which hadrons are emitted from the source is unknown and may be different from event to event. So we treat $a$ as a free parameter and discuss only fluctuations in hadron phase with $a<0$ in the following since in the quark phase only a few hadrons can be produced through fluctuations. From the distribution of Eq. (1) one gets the mean multiplicity for $a<0$

$$
\langle n\rangle=s \frac{H_{1}\left(|a| / B^{2},-\sqrt{s} B\right)}{H_{0}\left(|a| / B^{2},-\sqrt{s} B\right)},
$$

with $H_{n}(u, v) \equiv \int_{0}^{\infty} d y y^{n} \exp \left(-y^{3}+u v^{2} y+v y^{2}\right)$ and $s=\left(\delta^{2} / c\right)^{1 / 3}$ representing the bin width $\delta, B=b / \sqrt{c}$. This expression is different from that for second-order phase transition case in that the mean multiplicity in first-order case depends on three (instead of two) variables, the bin width $s$, the temperature $|a|$, and $B$. The mean multiplicity in a bin is proportional to $s$ at small phase space bin widths, thus it can be very small. For very small bins the distribution must be concentrated around $P_{0}$, and both $P_{q}$ and $p_{q}$ for $q>1$ must be very small. So a direct comparison between $P_{q}$ and $p_{q}$ could induce large uncertainty. This demands that the bin width in real experimental analysis should be large enough to ensure the mean multiplicity in the bin not too small (larger than 0.5, say). Of course, smaller bins can be used if a precise determination of both $P_{q}$ and $p_{q}$ can be obtained from data with high statistics. Naively, one may expect the distribution to approach to a Poisson one in the small $s$ limit. In the Ginzburg-Landau description for first-order phase transition, it is not the case. In the small $s$ limit, the mean multiplicity is approximately $s \Gamma(2 / 3) / \Gamma(1 / 3)$,

$$
P_{q} \simeq \frac{s^{q}}{q !} \frac{\Gamma\left(\frac{q+1}{3}\right)}{\Gamma(1 / 3)}, \quad p_{q} \simeq \frac{s^{q}}{q !}\left(\frac{\Gamma(2 / 3)}{\Gamma(1 / 3)}\right)^{q} .
$$

Thus except $P_{1}$, which approaches to $p_{1}$ in the small $s$ limit, $P_{q} \gg p_{q}$ for $q>1$ in the small $s$ limit. Because of the normalization of both $P_{q}$ and $p_{q}$, the dynamical factor $d_{q}$ must be larger than 1.0 for some $q$ and less than 1.0 for some other $q$ if there exist dynamical fluctuations. Using the Ginzburg-Landau description for first-order phase transition one can easily get

$$
d_{q}=\frac{H_{q}\left(\frac{|a|-1}{B^{2}},-\sqrt{s} B\right)}{H_{0}\left(|a| / B^{2},-\sqrt{s} B\right)} \frac{H_{0}^{q}\left(|a| / B^{2},-\sqrt{s} B\right)}{H_{1}^{q}\left(|a| / B^{2},-\sqrt{s} B\right)} \exp (\langle n\rangle) .
$$

To see the dependences of $\langle n\rangle$ and $d_{q}$ on the bin width $s$, we graph $\langle n\rangle$ and $\ln d_{q}$ as functions of $-\ln s$ in Fig. 1 for $B=-1.0,|a|=1.0$ and 2.0, respectively. One can see that for larger $s$ (or smaller $-\ln s$ ) the mean multiplicity is larger and that in $d_{q}$ is larger than $1.0\left(\ln d_{q}\right.$ larger than 0.0) for some $q$ but less than 1.0 for some other $q$. For smaller $s$ (or larger $-\ln s$ ) simple increasing behaviors can be seen for all $d_{q}$ with the increase of $-\ln s$. This means that the dynamical influence can be observed more easily in smaller windows in the kinetic regime. This can be 
understood once one notices the fact that different dynamical fluctuations may offset each other and become less obviously observable in large bins. In the limit of $s \rightarrow 0.0$ all $d_{q}$ approach to certain saturation values. Another feature for the dependences of $\langle n\rangle$ and $\ln d_{q}$ is that the detailed dependences rely on the temperature $|a|$, especially obvious in larger bin width $s$.

It is instructive to notice that the ratio $p_{q}(\langle n\rangle) / p_{1}(\langle n\rangle)$ is an increasing function of $\langle n\rangle$ for $q>1$, although $p_{q}(\langle n\rangle)$ itself exhibits complicated behavior with the increase of $\langle n\rangle$. Moreover there exists a scaling law between $p_{q}$

$$
\frac{p_{q}(\langle n\rangle)}{p_{1}(\langle n\rangle)}=\frac{2^{q-1}}{q !}\left(\frac{p_{2}(\langle n\rangle)}{p_{1}(\langle n\rangle)}\right)^{q-1}
$$

From this scaling law, one may conjecture that it is more interesting to study the dependence on $s$ of

$$
D_{q} \equiv \frac{d_{q}}{d_{1}}=\frac{P_{q} / P_{1}}{p_{q} / p_{1}}
$$

instead of $d_{q}$ and that one may expect similar scaling behavior between $D_{q}$ when the resolution is changed. One can see from below that it is true. Now we turn to study $D_{q}$ for first-order quark-hadron phase transition. If there is no dynamical reason, $P_{q}=p_{q}$, one can see that $D_{q}$ for all $q$ can have only one value, 1.0 , no matter how large or small the bin width is. So from the range of values $D_{q}$ takes, one can evaluate the strength of dynamical fluctuations. $D_{q}$ can be expressed in terms of $H_{n}(u, v)$ as

$$
\ln D_{q}=(q-1) \ln \frac{H_{0}\left(\frac{|a|}{B^{2}},-\sqrt{s} B\right)}{H_{1}\left(\frac{|a|}{B^{2}},-\sqrt{s} B\right)}+\ln \frac{H_{q}\left(\frac{|a|-1}{B^{2}},-\sqrt{s} B\right)}{H_{1}\left(\frac{|a|-1}{B^{2}},-\sqrt{s} B\right)} .
$$

Besides the bin width $s$, there is in last expression two more parameters $B$ and $|a|$ which is a measure of how far from the critical temperature the hadronization process occurs and is unknown in current experiments. First let us fix $B=-1.0$ and let $|a|=1.0$ and 2.0, respectively. The dependence of $D_{q}$ on $-\ln s$ is shown in the upper part in Fig. 2. Now $D_{q}$ for all $q$ is an increasing function of $-\ln s$. For small $-\ln s$ values of $D_{q}$ depend strongly on parameter $|a|$, but they approach parameter independent values for large $-\ln s$. The similarity among $D_{q}$ for different $q$ suggests that there may exist a scaling law between $D_{q}$ and $D_{2}$. The same data $D_{q}$ for $q>2$ are reshown in the lower part in Fig.2 as functions of $D_{2}$. Power scaling laws between $D_{q}$ and $D_{2}$ are found for both $|a|=1.0$ and 2.0. For other values of $|a|$ the similar power law dependence is checked to be true. Thus one has

$$
\ln D_{q}=A_{q}+B_{q} \ln D_{2}
$$

with $A_{q}$ and $B_{q}$ depending on $|a|$ for fixed parameter $B=-1.0$. The fitted results of $A_{q}$ and $B_{q}$ from curves in lower part in Fig. 2 are shown in Fig. 3 as functions of $\ln (q-1)$ for $|a|=1.0$ and 2.0. It is obvious that both $\ln A_{q}$ and $\ln B_{q}$ have linear dependence on $\ln (q-1)$ for fixed $|a|$. Especially, for studying power law, we investigate $B_{q}$ and find that

$$
B_{q}=(q-1)^{\gamma},
$$

with $\gamma$ depending on $|a|$ for fixed $B=-1.0$. For visualization, the linear fitting curves for $\ln B_{q}$ vs $\ln (q-1)$ are shown also in Fig. 3 for $|a|=1.0$ and 2.0. The slopes for $A_{q}$ is about twice those for $B_{q}$. The exponent $\gamma$ increases with increasing $|a|$. When $|a|$ is zero, corresponding to the case in which hadrons are produced exactly at the critical point, numerical results show that $\gamma$ is 0.912 for fixed $B=-1.0$. With the increase of $|a|$ at fixed $B, \gamma$ increases quickly. For sufficiently large $|a|$, when the difference between $|a|-1$ and $|a|$ can be neglected, corresponding to the case in which hadrons are produced at temperature far below the critical point, one finds that $D_{q} \rightarrow F_{q}$, with $F_{q}$ the scaled factorial moment in the same kinematic bin which is given in $[10,11]$ for first-order phase transition as

$$
F_{q}=H_{q}\left(\frac{|a|}{B^{2}},-\sqrt{s} B\right) H_{0}^{q-1}\left(\frac{|a|}{B^{2}},-\sqrt{s} B\right) H_{1}^{-q}\left(\frac{|a|}{B^{2}},-\sqrt{s} B\right) .
$$

Similar relation between $D_{q}$ and $F_{q}$ is also true in the small $s$ limit. In these limiting cases, the scaling of $D_{q}$ is equivalent to that for the scaled factorial moments $F_{q}$, and one can get the exponent $\gamma=1.4066$ for large $-\ln s$ [15] or large $|a|$. This exponent is a little different from those obtained from the study of the factorial moments for both first- and second-order phase transitions $[9,10,13,14]$ and of the multiplicity difference correlators for second-order phase transition [16]. The dependence of $\gamma$ on $|a|$ is shown in Fig. 4 for fixed $B=-1.0$. The dependence of the exponent $\gamma$ on parameter $B$ is also studied. As an example, we fix parameter $|a|=1.0$. It can be seen clearly from 
Eq. (7) that $\gamma$ is equal to 1.0 for parameter $B=0.0$ which corresponds to the tricritical case. Since we are discussing first-order phase transition, $B$ should be negative. For larger $-B$ the exponent $\gamma$ is larger, as shown in Fig. 5. For other fixed parameter $|a|$ similar dependence of $\gamma$ on $B$ can also be seen.

In conclusion, dynamical factors $d_{q}$ and their ratios $D_{q}$ are studied to describe features of dynamical fluctuations in first-order quark-hadron phase transition. In Ginzburg-Landau description for first-order quark-hadron phase transition it is found that $D_{q}$ obeys a power law, $D_{q} \propto D_{2}^{B_{q}}$, with $B_{q}=(q-1)^{\gamma}$. Both $d_{q}$ and $D_{q}$ can be obtained quite easily in experimental analysis. First one can get $P_{q}$ by counting up the number of events with exactly $q$ hadrons in the bin. $p_{q}$ is of Poisson type and can be calculated from the experimental mean multiplicity $\langle n\rangle$ in the bin. Simple algebras give $d_{q}$ and $D_{q}$. The existence of dynamical fluctuations can be confirmed if $d_{q}$ and $D_{q}$ can take values very different from 1.0. The range of values for $D_{q}$ is a measure for the strength of dynamical fluctuations. The scaling between $D_{q}$ and $D_{2}$ is a possible signal for the formation of QGP, because up to now no other dynamical reason is known to induce such a scaling. The study of $D_{q}$ should be carried out in real experimental analysis in the near future to see whether QGP has been formed in current high energy heavy-ion collisions. As tools to study dynamical fluctuations $d_{q}$ and $D_{q}$ introduced in this paper may also be interesting in experimental analysis of leptonic and hadronic interactions without quark-hadron phase transitions.

This work was supported in part by the NNSF, the SECF and Hubei NSF in China.

\section{References}

[1] Z. Koba, H. B. Nielsen and P. Olesen, Nucl. Phys. B49, 317(1972).

[2] Cai Xu and Liu Lianshou, Lettere al Nuovo Cimento, 37, 495(1983).

[3] R. Botet, M. Ploszajczak, and V. Latora, Phys. Rev. Lett. 78, 4593(1997).

[4] E. A. De Wolf, I.M. Dremin, W. Kittel, Phys. Reports 270, 1(1996).

[5] W. Ochs and J. Wosiek, Phys. Lett. B289, 159(1992); B304, 144(1993); Z. Phys. C68, 269(1995); Yu. Dokshitzer and I.

M. Dremin, Nucl. Phys. B402, 139(1993); Ph. Brax, J.-L. Meunier and R. Peschanski, Z. Phys. C62, 649(1994).

[6] W. Kittel, S. V. Chekanov, D. J. Mangeol and W. J. Metzger, presented at the XXVII Symposium on Multiparticle Dynamics, September 8-12, 1997 Frascati-Rome, Italy, for the L3 Collaboration (hep-ex/9712003).

[7] A. Bialas and R. Peschanski, Nucl. Phys. B273, 703(1986); B308, 857(1988).

[8] C. B. Yang and X. Cai, hep-ph/9712533, submitted to Phys. Rev. C.

[9] R. C. Hwa and M. T. Nazirov, Phys. Rev. Lett. 69, 741(1992); R. C. Hwa and J. Pan, Phys. Rev. C50, 383(1994); R. C. Hwa and J. Pan, Phys. Lett. B297, 35(1992).

[10] R. C. Hwa, Phys. Rev. D47, 2773(1993).

[11] X. Cai, C. B. Yang, and Z. M. Zhou, Phys. Rev. C54, 2775(1996).

[12] C. B. Yang, X. R. Wang and X. Cai, Science in China, A27, 624(1997) (in Chinese); A40, 1065(1997) (in English).

[13] R. C. Hwa, Phys. Rev. C50, 383(1994); I.A. Lebedev and M. T. Nazirov, Mod. Phys. Lett. A9, 2999(1994); L. F. Babichev, D. V. Klenitsky, V. I. Kuvshinov, Phys. Lett. B345, 269(1995).

[14] A. K. Mohanty and S. K. Kataria, Phys. Rev. Lett. 73, 2672(1994).

[15] C. B. Yang and X. Cai, hep-ph/9711329, submitted to J. Phys. G.

[16] C.B. Yang and X. Cai, Phys. Rev. C57, 2049(1998); R.C. Hwa, Phys. Rev. D57, 1831(1998).

\section{Figure Captions}

Fig. 1 Dependences of the mean multiplicity in a bin and the dynamical factor $d_{q}(q=1,2,3,4,5$, and 6$)$ on the bin width $-\ln s$ for fixed $B=-1.0,|a|=1.0$ and 2.0.

Fig. 2 Upper figure: Dependences of $D_{q}$ on bin width $-\ln s$ for $|a|=1.0$ and 2.0 and $B=-1.0$ for $q=2,3,4,5$, 6; Lower figure: Scaling behaviors between $D_{q}$ and $D_{2}$ for $B=-1.0,|a|=1.0$ and 2.0.

Fig. 3 Coefficients for the scaling between $D_{q}$ and $D_{2}, \ln D_{q}=A_{q}+B_{q} \ln D_{2}$, as functions of $\ln (q-1)$ for fixed $B=-1.0,|a|=1.0$ and 2.0. Linear fitting curves are shown for $B_{q}=(q-1)^{\gamma}$. 
Fig. 4 Dependence of exponent $\gamma$ on $|a|$ for fixed $B=-1.0$. For large $|a|, \gamma$ is about 1.4.

Fig. 5 Dependence of exponent $\gamma$ on $-B$ for fixed $|a|=1.0$ for first-order quark-hadron phase transition $(B<0)$. 


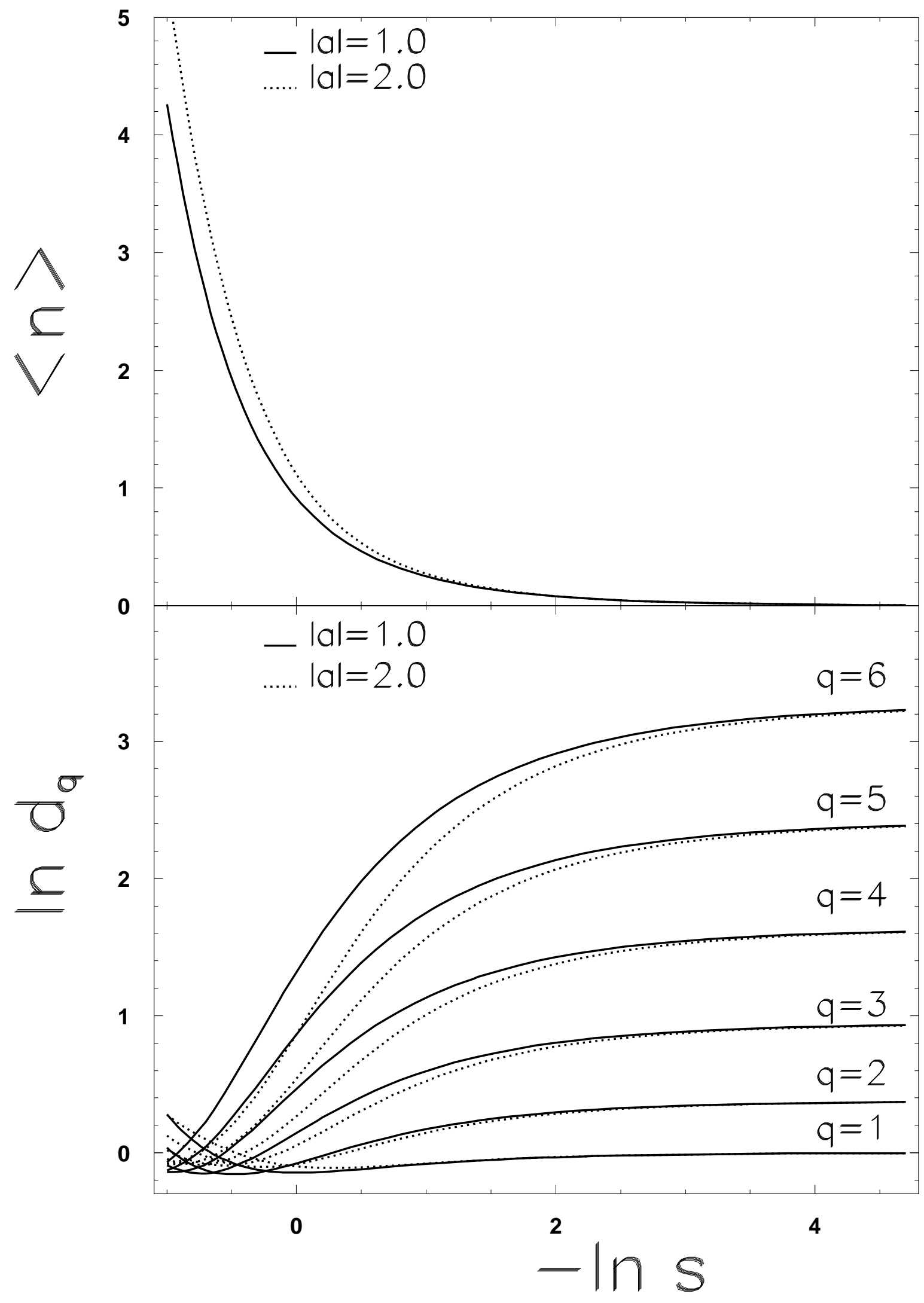



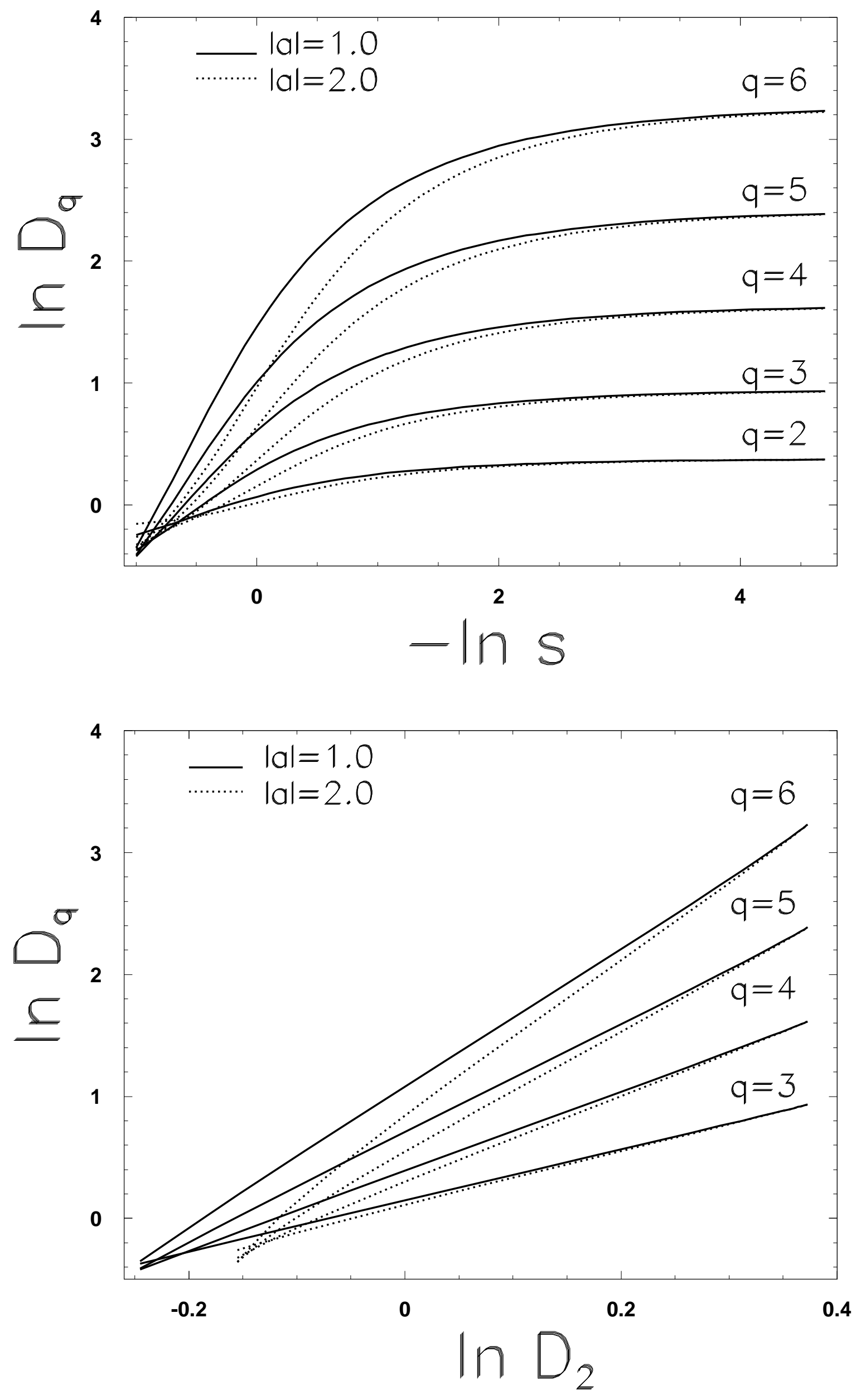


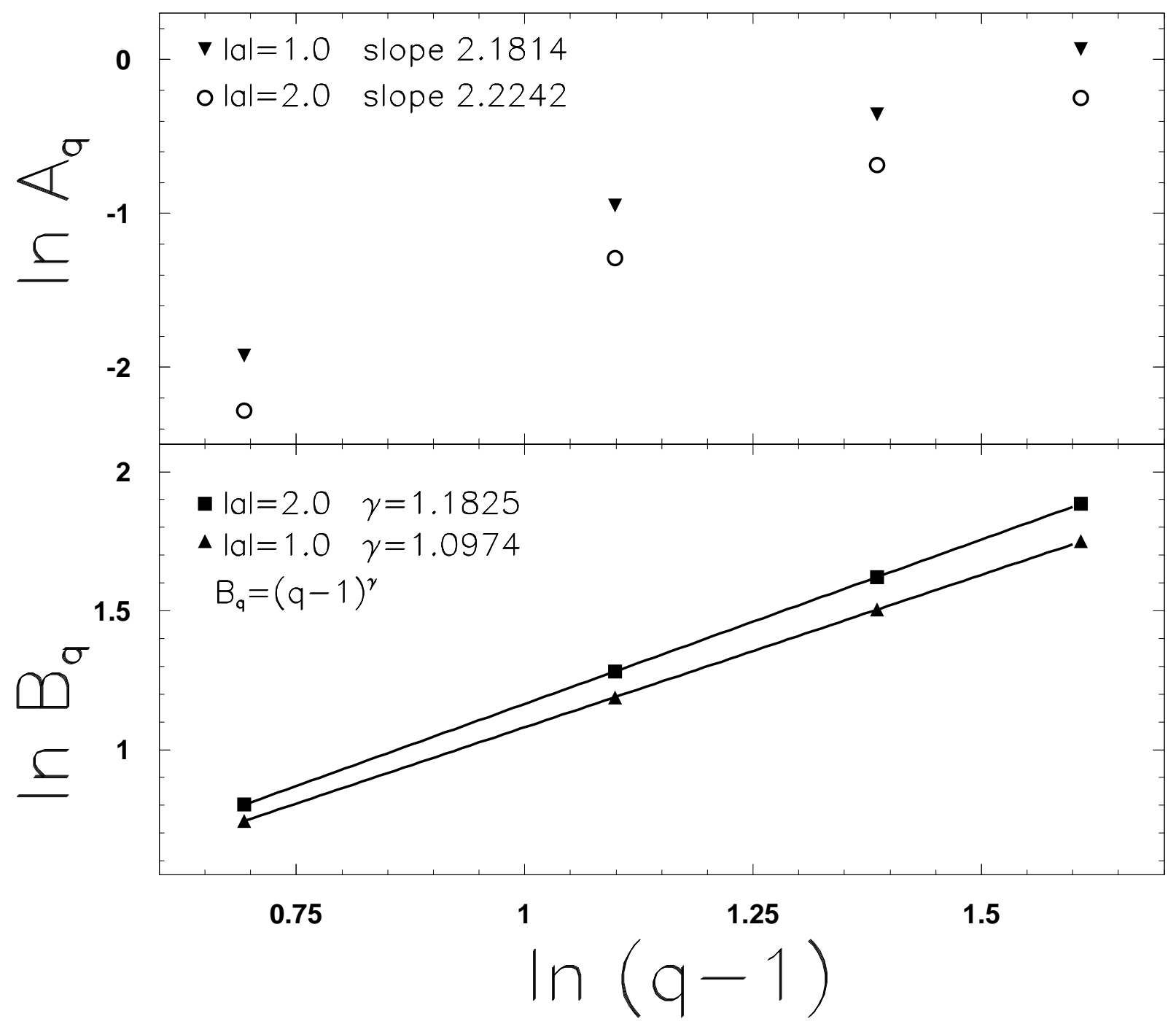




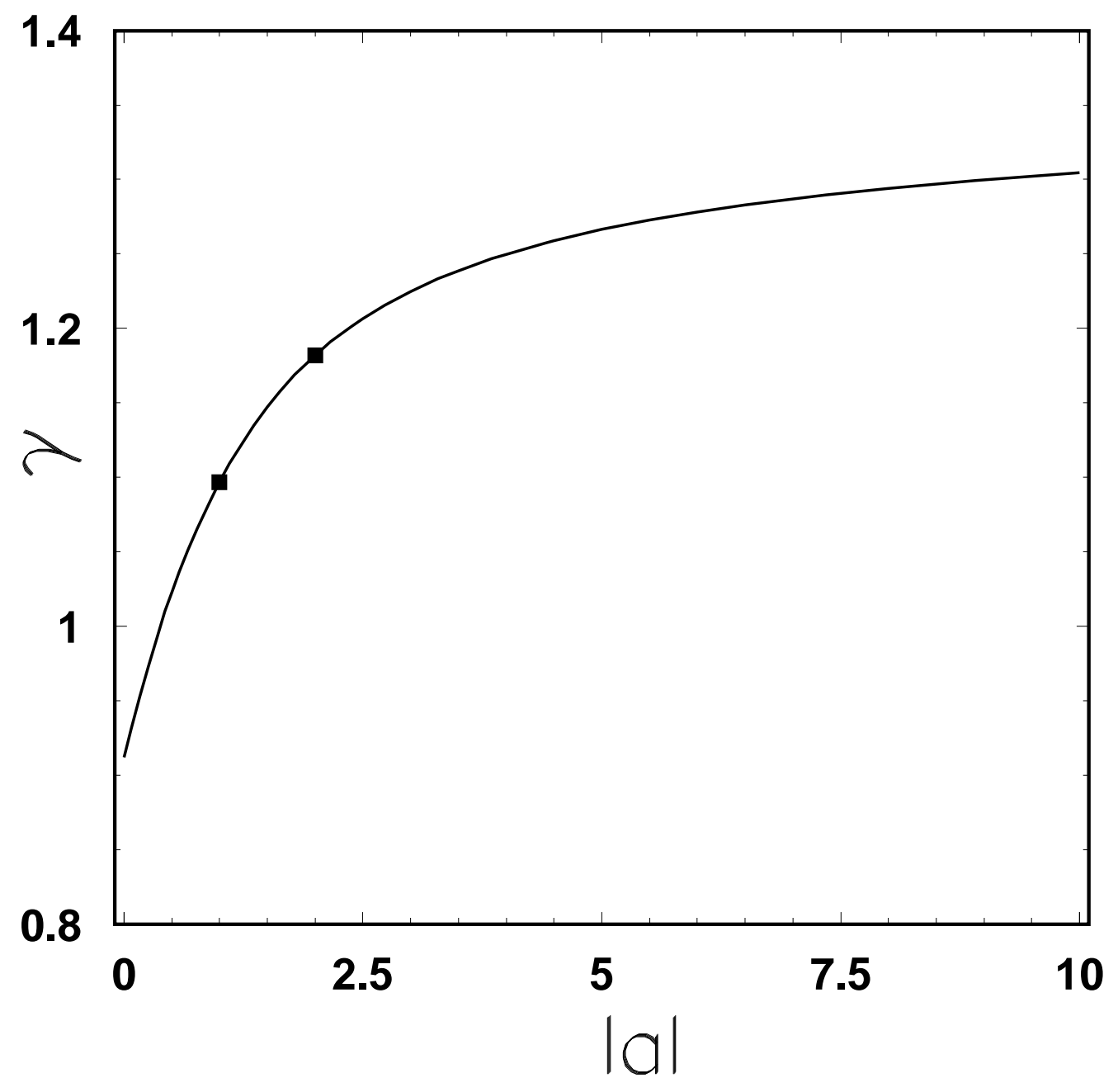




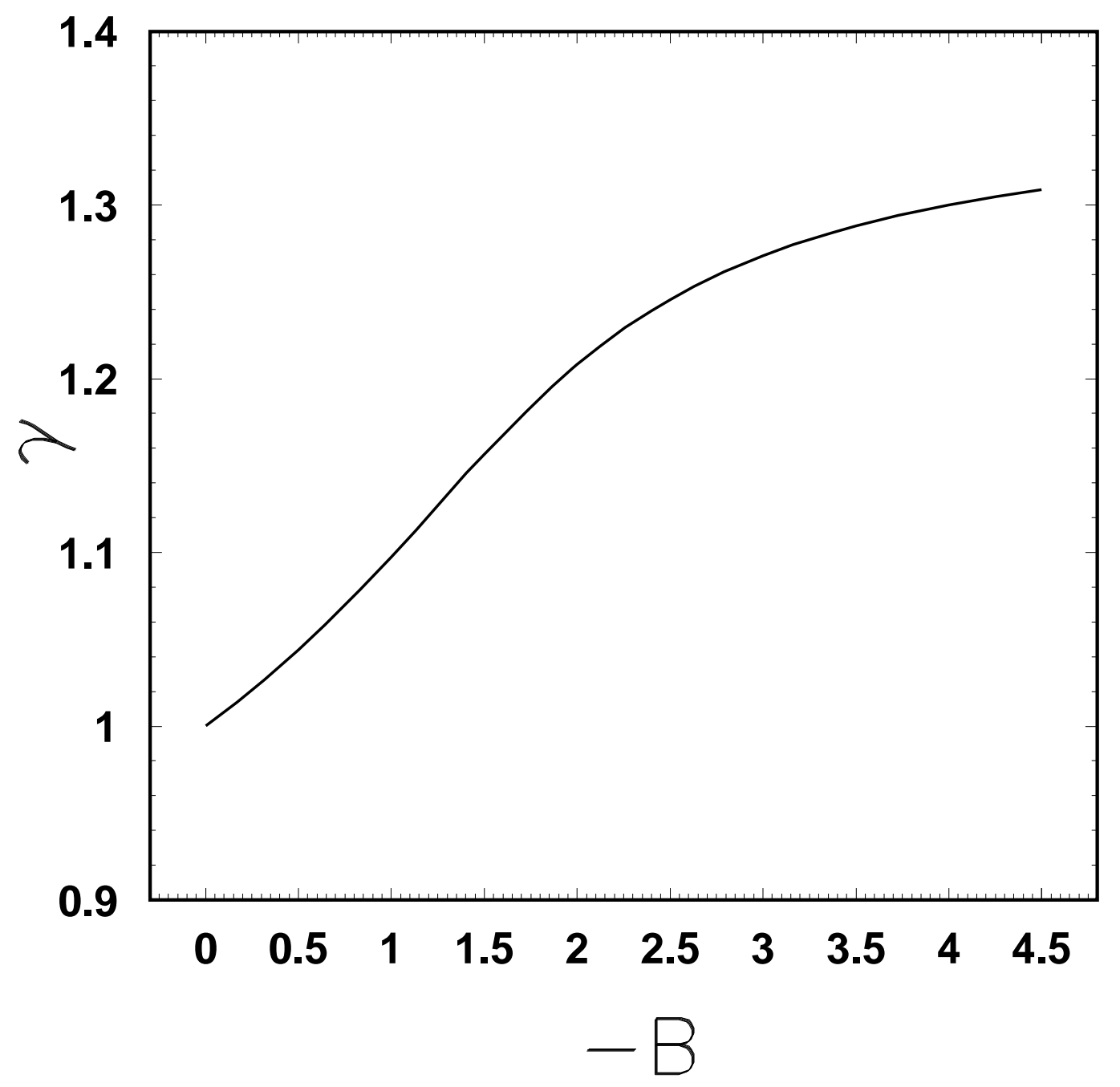

